
\title{
3 Research Suare \\ The Effect of Decision-aid Based Counseling on Cervical Cancer Screening Behavior Among Women: a Quasi-experimental Study
}

\section{Elnaz Malekzadeh}

Tabriz University of Medical Sciences

\section{Roghaiyeh Nourizadeh}

Tabriz University of Medical Sciences

Azizeh Farshbaf-Khalili

Tabriz University of Medical Sciences

Esmat Mehrabi ( $\nabla$ b.mehrabi62@gmail.com )

Tabriz University of Medical Sciences

\section{Sevil Hakimi}

Tabriz University of Medical Sciences

\section{Research Article}

Keywords: Cervical cancer screening, decision-aid, pap smear, SDM, stages of behavior change

Posted Date: May 6th, 2021

DOI: https://doi.org/10.21203/rs.3.rs-488862/v1

License: (c) (1) This work is licensed under a Creative Commons Attribution 4.0 International License. Read Full License 


\section{Abstract}

Background: The present study was designed to determine the effect of decision-aid-based counseling on changing cervical cancer screening behavior among women.

Methods: This quasi-experimental study was conducted on 154 women aged 21-59 with no pap smear history during the past three years. The participants were assigned to the intervention and control groups through randomized block design with block sizes of 4 and 6 and a 1:1 allocation ratio. The data were collected using the socio-demographic and midwifery questionnaire, stages of change checklist, and shared decision making (SDM) and DC questionnaire before and six months after the intervention by interview and then analyzed by SPSS 24 software. The chi-square, independent t-test, ANCOVA tests were used.

Results: A significant difference was observed between the two groups in terms of changing the stages of cervical cancer screening behavior six months after the intervention. After the intervention, the frequency of individuals entered the preparation or action stage was more than the control group $(P<0.001)$. The mean score of SDM in the intervention group was significantly higher than the control group [35.48 (1.18) vs. 27.56 (1.18) (Adjusted mean difference: 7.92; 95\% Cl: 5.59 to 10.25; $\mathrm{P}<0.001$ )]. The mean score of total DC in the intervention group was significantly lower than the control group [29.16 (1.09) vs. 34.14 (1.09) (Adjusted mean difference: $-4.97 ; 95 \% \mathrm{Cl}: 1.09$ to 8.04; $\mathrm{P}<0.001$ )].

Conclusions: It is suggested to apply decision-aid based counseling along with rutin trainins for promotimg the stages of cervical cancer screening behavior, increasing the , and reducing the DC regarding performing screening.

\section{Background}

Cervical cancer is the fourth most common cancer among women globally, especially in developing countries $[1,2]$. More than $80 \%$ of deaths due to cervical cancer occur in the low- and middle-income countries [3], which made the World Health Organization (WHO) call for the global control of cervical cancer in May 2018 to eradicate the disease [4] since this cancer is potentially one of the most preventable cancers [5].

The standard approaches for early detection of cervical cancer are screening women through cytology (Pap smear) and performing an HPV(Human Papilloma Virus) test (co-test). If the cytology is positive, the diagnosis of CIN (Cervix Insito Neoplasia) stages is confirmed based on the colposcopy and lesion sampling [6]. Early detection of cervical cancer through Pap smear is very important in reducing its complications and mortality [7]. However, the available evidence indicates the low level of women's knowledge about screening and early detection methods and their benefits for cervical cancer, which increases the need to design programs based on the education and promotion of women's knowledge in this field $[8,9]$. 
On the other hand, performing screening for cervical cancer is a decision that people make after receiving sufficient information about the method of testing and performing screening and its advantages and disadvantages. It is worth noting that choosing the best option is difficult for patients in many healthrelated decisions. The intervention programs to encourage women to perform screening influence the women's willingness to decide and subsequently change their behavior to participate in screening programs [10]. As stages of the change model address how informed decisions have been made, and the specified stages toward making a behavior change include pre-contemplation stage: I do not intend to do in the next six months (score 1), contemplation stage: I intend to do in the next six months (score 2), preparation stage: I intend to do in the next 30 days (score 3), action stage: I have done recently (score 4), and maintenance stage.

Selecting the best option in many health-related decisions is difficult for patients. This type of decision is regarded as "sensitive preferences," requiring considering the advantages and disadvantages of the options ahead [11]. Decision-aid helps individuals make informed decisions and perform or not perform behavior by providing evidence-based information and expressing the benefits and harms of health care and consequently, influences the DC (DC) or the satisfaction with the decision. It is worth noting that decision aid is designed as a tool to support and complement counseling, and it is not considered as a substitute for counseling [12]. Shared Decision Making (SDM) could facilitate the behavior.

SDM is defined as a process in which health options are selected by the patient and healthcare provider [13]. Further, SDM is considered the most important part of patient-centered care [14]. Obviously, as the consumers of health services, patients have rights such as individual care, respecting their beliefs and values, and knowing and participating in care decisions. In the same vein, the first condition for participating in decision-making is to have sufficient information about medical methods and procedures [15].

The results of a study that examined the use of SDM in Iran indicated that despite the legislation of the Ministry of Health regarding patient's informed consent, SDM is still rarely used in the health care delivery system. Further, it is suggested that further studies investigate the impact of SDM in Iran due to the lack of research in this field [16].

Given the low frequency of cervical cancer screening behavior, the interventions should be performed, and the effect of the interventions on changing women's screening behavior should be investigated based on the theory of behavior change, indicating a person's behavior change in stages. Therefore, considering the importance of cervical cancer screening in reducing mortality and in order to identify the appropriate intervention method to increase women's participation in the cervical cancer screening programs, the present study aimed to determine the effect of decision-aid-based counseling on changing women's screening behavior.

\section{Methods}




\section{Study design and participants}

This quasi-experimental study was conducted from July 2019 to December 2019. The study population were married women aged 21-59 who were referred to health centers in Tabriz, Iran. The inclusion criteria consisted of: married women aged 21-59 with no history of doing Pap smear in the past three years and no intention to do it in the next six months, no history of cervical cancer or a history of cervical cancer among first degree relatives, literacy, and having a telephone number for follow-up. The exclusion criteria included: pregnant women, history of participating in training and interventions programs related to cervical cancer screening, a history of spotting or bleeding after intercourse, or irregular uterine bleeding.

the change of stages of behavior six months after the intervention in the decision-aid and control groups as a primary outcome and the change of SDM and DC six months after the intervention in the decisionaid and control groups, as the secondary outcomes were considered.

\section{Sample Size}

Based on the study of Ezzati et al. [17] and the variable of cervical cancer screening behavior and considering $(P 1=0.55)$ with a $35 \%$ increase in the frequency caused by intervention $(P 2=0.75)$ and with respect to the power $=80 \%$, one-sided $a=0.05$ in G Power software, the sample size was calculated 70 subjects for each group, which increased to 77 , due to a $10 \%$ probability of sample loss.

\section{Sampling}

The sampling process was started after obtaining the permission of the Ethics Committee of Tabriz University of Medical Sciences (Code: IR.TBZMED.REC.1398.319). The city of Tabriz with 85 healthcare centers includes the health records of all married women aged $21-59$, such as telephone number and addresses.

Participants were selected from the most crowded healthcare centers in various areas with different socio-economic classes. The author visited the 15 selected centers and obtained data on married women aged 21-59 using the integrated health system (IHS), known as "SIB System". Then, she called eligible women, provided them with a brief description of the research objective and method, and obtained written informed consent from them to participate in the study.

\section{Randomization}

The participants were assigned to the intervention (decision-aid based counseling) and control groups using a randomized block design with block sizes of 4 and 6 and a 1:1 allocation ratio. A co-researcher, non-involved in the intervention and data analysis, generated the random allocation sequence by using Random Allocation software (RAS). The intervention type was written on a piece of paper and placed in opaque envelopes, numbered consecutively to conceal the allocation sequence. Envelopes from 1 to 154 were given to the study participants based on the entry order.

\section{Data collection tools}


Data collection tools included the socio-demographic and obstetric questionnaire, checklist of change stages, SDM, and DC questionnaire, which were completed through an interview by the first author. The socio-demographic questionnaire included items such as age, number of children, level of education, occupation, adequacy of household income, and midwifery history, including the number of pregnancies, age of first menstruation, age of menopause, age of first pregnancy, etc.

The checklist of change stages is designed based on the transtheoretical model (TTM) of behavior change [18]. The responses were scored based on a 5-point Likert scale as follows: pre-contemplation stage: I do not intend to do in the next six months (score 1), contemplation stage: I intend to do in the next six months (score 2), preparation stage: I intend to do in the next 30 days (score 3), action stage: I have done recently (score 4), and maintenance stage. The maintenance stage was not measured in the present study due to the time limit of the researcher. The tool's reliability was 0.71 using the Kappa index by measuring twice with an interval of two weeks [19].

\section{SDM}

The SDM scale was used to assess SDM among participants. A group of German researchers developed the original SDM questionnaire with 15 items in 2001, which was reduced to 11 items in the initial validity conducted in 2006 [20]. The final version included nine items, and the English version was used for validation in Iran. Participants answer each item using a 6-point Likert scale ranging from 0 (strongly disagree) to 5 (strongly agree). Finally, the total scores were calculated for each questionnaire, and the total scores (minimum 0 to maximum 45) of the questionnaire were determined to specify the perceived level of SDM. In a study conducted by Haj Ebrahimi et al. (2014), the Persian version of the questionnaire was validated, and the results demonstrated an acceptable level of reliability (Cronbach's alpha 0.901) and Pearson correlation coefficient was 0.70 , indicating a large and positive linear relationship between two variables[21].

DC

O'Conner's DC (DC) tool is a 16-item questionnaire with subscales of measuring uncertainty in the choice of options, factors influencing uncertainty (e.g., the sense of the lack of knowledge, lack of knowledge about personal values, and lack of support in decision making) and perceived impact of decision making (e.g., the sense of informed choice based on the value and the possibility of the implementation and expression of the satisfaction with choice) [13]. The questions are answered using a 5-point Likert scale ranging from 0 (completely agree) to 4 (completely disagree). All items' scores are summed, divided by 16, and multiplied by 25 to calculate the total score between $0-100$. The scores below 25 are associated with decision making, while the scores above 37.5 are related to delayed decision making or uncertainty about decision making. In addition, this scale contains five subscales, including Informed, value clarity, support, uncertainty, and effective decision. Higher scores on the scale or subscales indicate higher DC and less uncertainty, and less satisfaction with the choice [22]. In a study in Iran, Ghiasvandian et al. (2013) confirmed the questionnaire's psychometric properties. The tool was provided to ten patients with 
breast cancer, and the necessary changes were made for face validity. The Cronbach's alpha coefficient was reported 0.94 for the DC questionnaire [23].

\section{Intervention description}

The pre-test questionnaires, including socio-demographic and midwifery questionnaire, checklist of the stage of behavior change, and SDM questionnaire, and DC questionnaire, were completed through an interview by control and intervention groups (154 women). In the intervention group (77 people), a 60-90 minutes counseling session based on decision-aid content and routine training was held in the training room of the relevant health center by the Co-author author.

In the session, the author sought to establish good relationships with participants and gave them a feeling of assurance. The information on the prevalence of the disease and the risks of infection, the introduction of cervical cancer screening methods, and the advantages and disadvantages of performing and avoiding screening was provided during the in-person session. The possible advantages and disadvantages of cervix cancer screening methods (Inc. cervix cytology, HPV test, colposcopy) were presented, participants' questions were answered, and a decision-aid book was provided for reading at the end of the session. The content of the decision-aid booklet was prepared according to the booklet on the Ottawa site [24] and national guidelines [25] based entirely on up-to-date evidence of cervical cancer screening by the research team, and even how to perform screening was described by images. The contact number of the first author and corresponding author was written in the booklet for more information. The Control group just received routine training about cervix cancer screening.

The first telephone counseling session was provided by the author four weeks later for about ten min, to follow up and encourage them to carry out their questions about cervical cancer screening tests. The second session was held if participants tended and requested.

Six months after the intervention, the questionnaires were completed again by control and intervention groups by in-person interview. Adherence to the Pap-smear (action stage) was checked according to the health profile documented by health care providers. The decision-aid-based counseling was presented at the end of the study to the control group to comply with the ethical principles.

\section{Statistic analysis}

The SPSS (ver 24) statistical software was used for data analysis. The mean and standard deviation were reported normal in the quantitative variables. First, the normality of quantitative data was investigated using Kolmogorov-Smirnov, which indicated normal distribution for all data. The independent t-test and chi-square test were used to compare the socio-demographic characteristics between groups. The Chi-square test was used to compare the stages of change before the intervention, and ordinal regression was used after the intervention. In order to compare the mean score obtained from SDM and DC between groups, an independent t-test was used before the intervention and ANCOVA test 
with adjustment of baseline values was used after the intervention. The significance level was considered 0.05 .

\section{Results}

The present study was conducted on 154 women from April 2020 to June 2020 and follow-up continued until December 2020 (six-month follow-up). The mean (standard deviation) age of the intervention and control groups was 39.53 (10.1) and 38.44 (6.8), respectively. Most participants were housewives. There was no significant difference between the intervention and control groups in terms of the demographic and midwifery characteristics (Table 1 ). 
Table 1

The socio- demographic and midwifery characteristics

\begin{tabular}{|c|c|c|c|}
\hline Variable & $\begin{array}{l}\text { Intervention group } \\
(n=77)\end{array}$ & $\begin{array}{l}\text { Control group } \\
(n=77)\end{array}$ & $\mathbf{P}$ \\
\hline & $N(\%)$ & $\mathbf{N}(\%)$ & \\
\hline Age (years) * & $39.53(10.1)$ & $38.44(6.8)$ & $* \star 0.436$ \\
\hline Spouse age * & $46.10(9.5)$ & 44.25 (9.1) & $\star * 0.219$ \\
\hline Marriage age* & $21.64(3.4)$ & $20.84(4.7)$ & $* \star 0.239$ \\
\hline Education & & & $0.616_{c}$ \\
\hline Under diploma & $26(33.8)$ & $25(32.5)$ & \\
\hline Diploma & $36(46.8)$ & $32(41.6)$ & \\
\hline University & $15(19.5)$ & $20(26.0)$ & \\
\hline Spouse education & & & $0.817_{\zeta}$ \\
\hline Illiterate & $5(6.5)$ & $4(5.2)$ & \\
\hline Under diploma & $19(24.7)$ & $24(31.2)$ & \\
\hline Diploma & $18(23.4)$ & $18(23.4)$ & \\
\hline University & $35(45.5)$ & $31(40.3)$ & \\
\hline Employment status & & & $0.469_{C}$ \\
\hline Housewife & $46(59.7)$ & $49(63.6)$ & \\
\hline Employed & $20(26.0)$ & $14(18.2)$ & \\
\hline Other & $11(14.3)$ & $14(18.2)$ & \\
\hline Spouse employment status & & & $0.687_{C}$ \\
\hline Unemployed & $6(7.8)$ & $10(13.0)$ & \\
\hline Worker & $19(24.7)$ & $20(26.0)$ & \\
\hline Employee & $30(39.0)$ & $25(32.5)$ & \\
\hline Other & $22(28.6)$ & $22(28.6)$ & \\
\hline Family income level & & & $0.712_{\hat{\zeta}}$ \\
\hline
\end{tabular}




\begin{tabular}{|c|c|c|c|}
\hline Variable & $\begin{array}{l}\text { Intervention group } \\
(n=77) \\
N(\%)\end{array}$ & $\begin{array}{l}\text { Control group } \\
(n=77) \\
N(\%)\end{array}$ & $\mathbf{P}$ \\
\hline Not enough & $11(14.3)$ & $8(10.4)$ & \\
\hline Somewhat enough & $41(53.2)$ & $45(58.4)$ & \\
\hline Enough & $25(32.5)$ & $24(31.2)$ & \\
\hline Insurance status & & & $0.089_{\zeta}$ \\
\hline Yes & $68(88.3)$ & $59(76.6)$ & \\
\hline No & $9(11.7)$ & $18(23.4)$ & \\
\hline Suffering from genital warts & & & $1.000_{c}$ \\
\hline Yes & $3(3.9)$ & $4(5.2)$ & \\
\hline No & $74(96.1)$ & $73(94.8)$ & \\
\hline Number of deliveries & & & $0.196_{\zeta}$ \\
\hline Without delivery & $1(1.3)$ & $4(5.2)$ & \\
\hline 1 and 2 & $60(77.9)$ & $63(81.8)$ & \\
\hline More than 2 & $16(20.8)$ & $10(13.0)$ & \\
\hline
\end{tabular}

A total of 380 women were assessed, of whom 216 eligible individuals aged 21-59 without a history of doing cervix cytology in the last three years were enrolled. Forty-one individuals were excluded due to not having eligibility criteria (poor educational attainments or illiteracy $(n=41)$, having post-coital bleeding or abnormal uterine bleeding $(n=21)$. Finally, 154 women were allocated in to tow group randomized. Among 154 included participants (77 in each group), two individuals were withdrawn from control group (unwillingness to cooperate, $n=2$ ), and one other was withdrawn from the intervention group (unwillingness to cooperate, $n=1$ ) (Fig. 1).

Before the intervention, the comparison of the stages of behavior change in cervical cancer screening demonstrated that 57 women (74.0\%) were in the pre-contemplation stage and $20(26.0 \%)$ were in the contemplation stage in the intervention group. In the intervention group, 22 subjects $(28.6 \%)$ entered the contemplation stage, $15(19.5 \%)$ entered the preparation stage, and $19(24.7 \%)$ entered the action stage $(P<0.0001)$ after the intervention (Table 2$).$ 
Table 2

The frequency distribution and the comparison of behavior change in performing Pap smear in the intervention and control groups, before and 6month after the intervention

\begin{tabular}{|c|c|c|c|c|c|c|}
\hline \multirow{3}{*}{$\begin{array}{l}\text { Stages of behavior change in } \\
\text { performing } \\
\text { a Pap smear }\end{array}$} & \multicolumn{3}{|c|}{ Before intervention } & \multicolumn{3}{|c|}{ After the intervention } \\
\hline & Intervention & Control & $\mathrm{P}^{*}$ & Intervention & Control & $P^{\star \star}$ \\
\hline & $\mathrm{N}(\%)$ & $\mathrm{N}(\%)$ & & $\mathrm{N}(\%)$ & $\mathrm{N}(\%)$ & \\
\hline Pre-contemplation stage & $57(74.0)$ & $\begin{array}{l}60 \\
(77.9)\end{array}$ & \multirow[t]{4}{*}{0.736} & $21(27.3)$ & $\begin{array}{l}52 \\
(67.5)\end{array}$ & \multirow[t]{4}{*}{$\begin{array}{l}< \\
0.001\end{array}$} \\
\hline Contemplation stage & $20(26.6)$ & $\begin{array}{l}17 \\
(22.1)\end{array}$ & & $22(28.6)$ & $\begin{array}{l}13 \\
(16.9)\end{array}$ & \\
\hline Preparation stage & $0(0.0)$ & $0(0.0)$ & & 15 (19.5) & $5(6.5)$ & \\
\hline Action stage & $0(0.0)$ & $0(0.0)$ & & 19 (24.7) & $7(9.1)$ & \\
\hline
\end{tabular}

The mean score of SDM of the intervention group was significantly higher than that of the control group (Adjusted mean difference: 7.92 with 95\% confidence interval: 5.59 to $10.25(p<0.001)$ ). Further, the mean score of DC of the intervention group was significantly lower than that of the control group (Adjusted mean difference: -4.97 with 95\% confidence interval: 8.04 to $1.09(p<0.001)$ ), (Table 3). 
Table 3

The comparison of mean (SD) of SDM and decision conflict in the intervention and control groups, before and 6month after the intervention

\begin{tabular}{|c|c|c|c|c|}
\hline \multirow[t]{2}{*}{ Variable } & Intervention & Control & Mean difference & \multirow[t]{2}{*}{$\mathbf{P}$} \\
\hline & Mean (SD) & Mean (SD) & $95 \%$ confidence interval & \\
\hline \multicolumn{5}{|c|}{$\operatorname{SDM}(0$ to 45$)$} \\
\hline Before & $20.67(5.62)$ & $23.38(6.19)$ & $-2.71(-6.47$ to 1.05$)$ & $0.156^{*}$ \\
\hline After & $35.48(1.18)$ & $27.56(1.18)$ & 7.92 (5.59 to 10.25$)$ & $<0.001^{\star \star}$ \\
\hline \multicolumn{5}{|c|}{ Decisional conflict (Total)\# } \\
\hline Before & 42.07(4.92) & $40.01(3.71)$ & $2.06(-1.38$ to 5.51$)$ & $0.238^{*}$ \\
\hline After & $29.17(1.09)$ & $34.14(1.09)$ & $-4.97(-1.04$ to -8.09$)$ & $<0.001^{* *}$ \\
\hline \multicolumn{5}{|c|}{ Informed sub scale } \\
\hline Before & $43.89(5.11)$ & $41.21(5.79)$ & 2.68 (-1.67 to 4.35$)$ & $0.442^{*}$ \\
\hline After & $27.50(0.69)$ & $34.14(0.69)$ & $-6.64(-4.19$ to -9.09$)$ & $<0.001^{* *}$ \\
\hline \multicolumn{5}{|c|}{ Value Clarity sub scale } \\
\hline Before & $42.68(5.17)$ & $40.01(6.75)$ & $1.98(-1.34$ to 5.30$)$ & $0.645^{\star}$ \\
\hline After & $28.69(2.19)$ & $33.25(2.19)$ & $-4.56(-8.04$ to -1.09$)$ & $<0.001^{\star *}$ \\
\hline \multicolumn{5}{|c|}{ Support sub scale } \\
\hline Before & 41.57(3.54) & $40.11(4.33)$ & $1.46(-1.18$ to 4.11$)$ & $0.222^{*}$ \\
\hline After & $32.67(2.32)$ & $38.33(1.76)$ & $-5.66(-9.00$ to -2.33$)$ & $<0.001^{\star *}$ \\
\hline \multicolumn{5}{|c|}{ Uncertainty sub scale } \\
\hline Before & $42.40(4.12)$ & $40.00(3.41)$ & $2.40(-2.11$ to 4.51$)$ & $0.128^{*}$ \\
\hline After & $27.10(2.91)$ & $31.90(2.12)$ & $-4.80(-6.32$ to -3.28$)$ & $<0.001^{* *}$ \\
\hline \multicolumn{5}{|c|}{ Effective decision sub scale } \\
\hline Before & $42.83(6.32)$ & $41.9(5.89)$ & $1.93(-2.14$ to 6.00$)$ & $0.458^{*}$ \\
\hline After & $25.96(1.09)$ & $31.02(1.09)$ & $-5.05(-7.75$ to -2.36$)$ & $<0.001^{\star *}$ \\
\hline
\end{tabular}




\section{Discussion}

The results of the present study indicated that following decision-aid based counseling in the intervention group, 15 (19.5) subjects were in the stage of preparation for doing Pap smear and 19 (24.7) were in the action stage. While in the control group, 5 (6.5) women were in the preparation stage, and 7 (9.1) entered the action stage. The findings of the present study are significantly in line with the study results of Van agt et al. (2012), similarly reported a higher intention to participate in the breast cancer screening programs in the group received decision-aid based counseling [26].Akbari et al. (2019) examined the effect of decision-aid on the stages of the behavior change of women in the programs of breast cancer screening. They found that the frequency of women who participated in the breast screening increased in intervention group compared to the control group received the usual training [27]. Further, Suzanne Miller et al. (2005) conducted a study on 279 women at the Atlantic Regional Cancer Care Center, entitled "facilitating informed decision-making about breast cancer risk and genetic counseling using decision-aid based counseling". They concluded that the women received decision-aid based counseling were more likely to enter the stage of the contemplation to do genetic testing [28].

In the present study, the mean score of SDM in the intervention group was significantly higher than that of the control group, and women in the intervention group had a sense of participation in decisionmaking, which is consistent with the study results of Jayoung Han (2018) indicated that the use of the SDM approach and the relationship between health care providers and clients increase the willingness to perform screening [29].

In addition, some studies [30-32] examined the impact of SDM on cancer-related preventive care. The results indicated the participants' satisfaction with healthcare provider's and physicians' participation in the decision-making process for preventative measures and even their cancer treatment decisions.

Further, these studies investigated the effect of decision-aid on SDM, improving knowledge, and reducing DC among patients. In the present study, the mean score of DC in the intervention group was lower than that of the control group. Shapira et al. (2007) investigated the effect of web-based decision-aid on the menopausal hormone therapy (MHT) decision among 177 patients in the United States. They reported a significant difference between the group that received decision-aid and the control group about the DC [33], which is consistent with the result of the present study. Similarly, Kelly et al. (2017) evaluated the effect of decision aid among women aged 25-60, who were genetically predisposed to breast cancer. The results indicated that providing decision aid to guide patients for genetic testing to prevent breast cancer reduced the DC among women receiving decision aid [34].

Applying random allocation, the allocation concealment of the participants and relatively high sample size with different socio-demographic features were the strengths of the present study. The impossibility of financing the screening costs and the lack of insurance support were among the factors influencing women's decision-making for screening, which was one of the limitations of the present study.

\section{Conclusion}


The present study's findings indicated the effect of decision-aid-based counseling on promoting SDM and reducing DC about cervical cancer screening, resulting in the informed decision-making and behavior change for performing screening. Therefore, it is recommended that midwives working in healthcare centers use decision-aid based counseling for cervical cancer screening as a complementary method along with routine training. Also, further research is required regarding efficacy of DA without counselling in different contexts in clinical practice.

\section{Declarations}

\section{Ethics approval and consent to participate}

The study was approved by the ethics committee at the Tabriz University of Medical Sciences [Ethic code: IR.TBZMED.REC: 1398.319]. All participants signed a written informed consent form prior to their enrollment. Consent for publication not applicable.

\section{Availability of data and materials}

The datasets used and/or analysed during the current study available from the corresponding author on reasonable request.

\section{Conflict of interest}

The authors declare that they have no competing interests.

\section{Funding}

This study was funded by the Research Deputy of Tabriz University of Medical Sciences [grant Number: TBZMED.REC: 1398.319]. The study was undertaken as part of the project "effect of SDM on cx screening self-efficacy," a program to develop and evaluate comprehensive Decision support services for women in gynecology clinics. None of the funding bodies participated in the study's design, in the collection, analysis, and interpretation of the data, or the writing of the manuscript

\section{Authors' contributions}

EMZ and EM conducted an intervention. All authors contributed to designing the study. AFKH and SH analyzed the data. EM drafted the manuscript in collaboration with co-authors (EMZ, AFKH, RN, SH) and was responsible for the overall management of the study. All authors critically revised the manuscript and read and approved its final version.

\section{Acknowledgments}

The researcher hereby expresses her gratitude to the Department of Nursing and Midwifery in Tabriz University of Medical Sciences, all involved healthcare centers, clinics and their staff, and the women who participated in this study. 


\section{References}

1. Arbyn M, Weiderpass E, Bruni L, de Sanjosé S, Saraiya M, Ferlay J, et al. Estimates of incidence and mortality of cervical cancer in 2018: a worldwide analysis. The Lancet Global Health 2020;8(2):e191e203.

2. World Health Organization. Cervical cancer Geneva: WHO; 2018 [cited 2018]. Available from: http://www.who.int/cancer/prevention/diagnosis-screening/cervical-cancer/en/.

3. Vaccarella S, Laversanne M, Ferlay J, Bray F. Cervical cancer in A frica, L atin A merica and the C aribbean and A sia: Regional inequalities and changing trends. International Journal of Cancer2017;141(10):1997-2001.

4. Canfell K. Towards the global elimination of cervical cancer. Papillomavirus Research. 2019;8:100170.

5. Everett T, Bryant A, Griffin MF, Martin-Hirsch PP, Forbes CA, Jepson RG. Interventions targeted at women to encourage the uptake of cervical screening. The Cochrane database of systematic reviews. 2011(5):Cd002834. Epub 2011/05/13. doi: 10.1002/14651858.CD002834.pub2. PubMed PMID: 21563135; PubMed Central PMCID: PMCPmc4163962.

6. World Health Organization. WHO guidelines for screening and treatment of precancerous lesions for cervical cancer prevention: World Health Organization; 2013.

7. Saslow D, Runowicz CD, Solomon D, Moscicki AB, Smith RA, Eyre HJ, et al. American Cancer Society guideline for the early detection of cervical neoplasia and cancer. CA: a cancer journal for clinicians. 2002;52(6):342-62.

8. Farzaneh E, Heydari H, Shekarchi AA, Kamran A. Breast and cervical cancer-screening uptake among females in Ardabil, northwest Iran: a community-based study. OncoTargets and therapy. 2017;10:985.

9. Javanmanesh F, Dadkhah F, Zarepour N. Knowledge and awareness among Iranian women regarding the pap smear. Medical Journal of The Islamic Republic of Iran (MJIRI). 2008;22(2):68-73.

10. Spadea T, Bellini S, Kunst A, Stirbu I, Costa G. The impact of interventions to improve attendance in female cancer screening among lower socioeconomic groups: a review. Preventive medicine. 2010;50(4):159-64. Epub 2010/01/23. doi: 10.1016/j.ypmed.2010.01.007. PubMed PMID: 20093138.

11. Evidence C. How much do we know? 2013 [cited 29 October 2013]. Available from: clinicalevidence.bmj.com/x/set/static/cms/efficacy-categorisations.html.

12. Ottawa Hospital Research Institute. Patient Decision Aids ottawa2018. Available from: https://decisionaid.ohri.ca/.

13. Makoul G, Clayman ML. An integrative model of shared decision making in medical encounters. Patient education and counseling. 2006;60(3):301-12. Epub 2005/07/30. doi: 10.1016/j.pec.2005.06.010. PubMed PMID: 16051459.

14. Weston W. Informed and shared decision-making: the crux of patient-centered care. CMAJ : Canadian Medical Association journal = journal de l'Association medicale canadienne. 2001;165(4):438-9. 
Epub 2001/09/04. PubMed PMID: 11531054; PubMed Central PMCID: PMCPmc81370.

15. World Health Organization. Should patient decision aids (PtDAs) be introduced in the health care system World Health Organization Regional Office for Europe2005.

16. Rahimi SA, Alizadeh M, Légaré F. Shared decision making in Iran: Current and future trends. Zeitschrift für Evidenz, Fortbildung und Qualität im Gesundheitswesen. 2017;123:52-5.

17. Ezzati E, Shariat F, Moradi F. The effect of a blended educational program (BEP) on cervical cancer screening behavior among housekeeper women in West Eslamabad in 2016: An application of health belief model. scientific journal of ilam university of medical sciences. 2017;25(1):110-20.

18. Peck A. Changing the face of standard nursing practice through telehealth and telenursing. Nursing administration quarterly. 2005;29(4):339-43.

19. Ghahramani M, Moodi M, Alami A, Moasheri N. STAGES OF PAP SMEAR SCREENING CHANGE BEHAVIOR BASED ON TTM IN WOMEN REFERRING TO HEALTH CENTERS IN GONABAD-YEARS 1393. 2015.

20. Simon D, Schorr G, Wirtz M, Vodermaier A, Caspari C, Neuner B, et al. Development and first validation of the shared decision-making questionnaire (SDM-Q). Patient education and counseling. 2006;63(3):319-27.

21. Ebrahimi MAH, Hajebrahimi S, Mostafaie H, Pashazadeh F, Hajebrahimi A. Physicians' perspectives toward shared decision making in developing countries. British Journal of Medicine and Medical Research. 2014;4(18):3458.

22. O'Connor AM. Validation of a DC scale. Medical decision making. 1995;15(1):25-30.

23. Ghiyasvandian S, Dehghan Nayeri N, Haghani H. The Effect of Decision Aid's Pakage in Selected Treatment by Patients with Early Stage Breast Cancer and Decision making Outcomes. Journal of Fasa University of Medical Sciences. 2013;3(3):271-9.

24. Patient decision aids, cervical cancer screening. Available frome: https://decisionaid.ohri.ca/AZsearch.php?criteria=screening. 2021/1/10.

25. Ahmad Koosha, Ali Ghanbari Motlagh, Ahdieh Maleki, Mehdi Najmi, Mahboubeh Dini, Masoumeh Arjmandpour, et al. Collection of Basic Interventions for Non-Communicable Diseases in the Primary Health Care System of Iran (Irapen). 2017. Executive Instruction and Educational Content of Midwives. . Ministry of Health, Medical Education, available from: https://baharestaniumsacir/files/baharestan/files/FixMaMapdf. 2021/1/3.

26. van Agt H, Fracheboud J, van der Steen A, de Koning H. Do women make an informed choice about participating in breast cancer screening? A survey among women invited for a first mammography screening examination. Patient education and counseling. 2012;89(2):353-9.

27. Akbari Z, Mehrabi E, Mirghafourvand M, Nourizadeh R. The effect of decision aid on breast cancer screening behaviors based on theory of stage of change: an interventional study. Crescent Journal of Medical and Biological Sciences. 2020;7(2).

28. Miller SM, Fleisher L, Roussi P, Buzaglo JS, Schnoll R, Slater E, et al. Facilitating informed decision making about breast cancer risk and genetic counseling among women calling the NCl's Cancer 
Information Service. Journal of Health Communication. 2005;10(S1):119-36.

29. Han J, Jungsuwadee P, Abraham O, Ko D. Shared Decision-Making and Women's Adherence to Breast and Cervical Cancer Screenings. International Journal of Environmental Research and Public Health. 2018;15(7):1509.

30. Kehl KL, Landrum MB, Arora NK, Ganz PA, Van Ryn M, Mack JW, et al. Association of actual and preferred decision roles with patient-reported quality of care: shared decision making in cancer care. JAMA oncology. 2015;1(1):50-8.

31. Mead EL, Doorenbos AZ, Javid SH, Haozous EA, Alvord LA, Flum DR, et al. Shared decision-making for cancer care among racial and ethnic minorities: a systematic review. American journal of public health. 2013;103(12):e15-e29.

32. Coulter A. Patient information and shared decision-making in cancer care. British Journal of Cancer. 2003;89(1):S15-S6.

33. Schapira MM, Gilligan MA, McAuliffe T, Garmon G, Carnes M, Nattinger AB. Decision-making at menopause: a randomized controlled trial of a computer-based hormone therapy decision-aid. Patient education and counseling. 2007;67(1-2):100-7.

34. Metcalfe KA, Dennis C-L, Poll A, Armel S, Demsky R, Carlsson L, et al. effect of decision aid for breast cancer prevention on DC in women with a BRCA1 or BRCA2 mutation: a multisite, randomized, controlled trial. Genetics in Medicine. 2017;19(3):330-6.

\section{Figures}




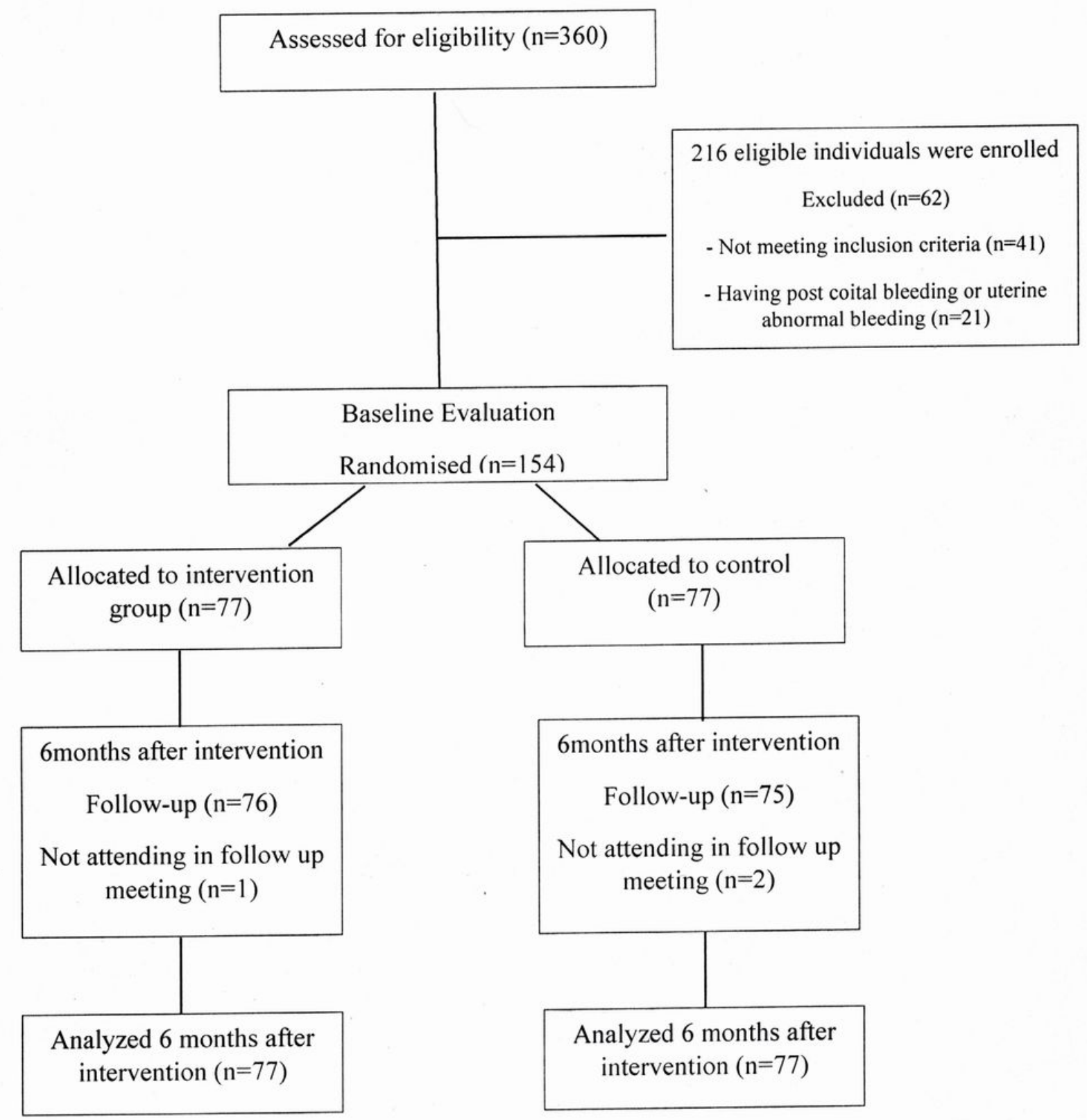

Figure 1

Flow chart 